\title{
Estimation of the interference coupling into cables within electrically large multiroom structures
}

\author{
J. Keghie ${ }^{1}$, R. Kanyou Nana ${ }^{1}$, B. Schetelig ${ }^{1}$, S. Potthast ${ }^{2}$, and S. Dickmann ${ }^{1}$ \\ ${ }^{1}$ Helmut-Schmidt-University/University of the Federal Armed Forces Hamburg, Germany \\ ${ }^{2}$ Bundeswehr Research Institute for Protective Technologies and NBC-Protection, Munster, Germany
}

\begin{abstract}
Communication cables are used to transfer data between components of a system. As a part of the EMC analysis of complex systems, it is necessary to determine which level of interference can be expected at the input of connected devices due to the coupling into the irradiated cable. For electrically large systems consisting of several rooms with cables connecting components located in different rooms, an estimation of the coupled disturbances inside cables using commercial field computation software is often not feasible without several restrictions. In many cases, this is related to the non-availability of computing memory and processing power needed for the computation.

In this paper, we are going to show that, starting from a topological analysis of the entire system, weak coupling paths within the system can be can be identified. By neglecting these coupling paths and using the transmission line approach, the original system will be simplified so that a simpler estimation is possible. Using the example of a system which is composed of two rooms, multiple apertures, and a network cable located in both chambers, it is shown that an estimation of the coupled disturbances due to external electromagnetic sources is feasible with this approach. Starting from an incident electromagnetic field, we determine transfer functions describing the coupling means (apertures, cables). Using these transfer functions and the knowledge of the weak coupling paths above, a decision is taken regarding the means for paths that can be neglected during the estimation. The estimation of the coupling into the cable is then made while taking only paths with strong coupling into account. The remaining part of the wiring harness in areas with weak coupling is represented by its input impedance. A comparison with the original network shows a good agreement.
\end{abstract}

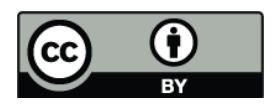

Correspondence to: J. Keghie (keghie@hsu-hh.de)

\section{Introduction}

The determination of induced disturbances in electrical systems cabling is of great importance in the industrial area. This is particularly true for electrically large systems like ships where many extended cable bundles run through the structure. In Kanyou Nana et al. (2008) a method was presented, that allows the identification of the contribution of the different coupling paths (apertures and cables) to the overall response of electronics located in one room, when irradiated by an Intentional Electromagnetic Interference (IEMI) source. In this paper we use the topological modeling procedure (Baum, 1982) and also take advantage of works done by Parmantier and Degauque (1996) to investigate a scenario that is common in real systems like ships. Those systems are characterized by a large amount of rooms and also many cable bundles connecting several components located in different rooms. We illustrate our approach using a two-room structure as shown in Fig. 1. The structure of this paper is as follows: in Sect. 2 we present the system to be investigated. Then we illustrate our approach and deal with criteria for the classification of weak and strong coupling paths in Sect. 3, where we also present our estimation procedure with the relevant theoretical background. After that, we apply the procedure to a system and discuss the result in Sect. 4. Section 5 gives a summary of the paper.

\section{Problem description}

The geometry we use for our investigation is illustrated in Fig. 2. The geometry consists of two interconnected rooms with a test network of total length $l$ connecting electronic components modeled by their input impedances $Z_{1}$ and $Z_{2}$ respectively. $Z_{1}$ is located in room 1 and $Z_{2}$ in room 2 . The structure has two apertures to the external environment and one common aperture between the two rooms. The test

Published by Copernicus Publications on behalf of the URSI Landesausschuss in der Bundesrepublik Deutschland e.V. 


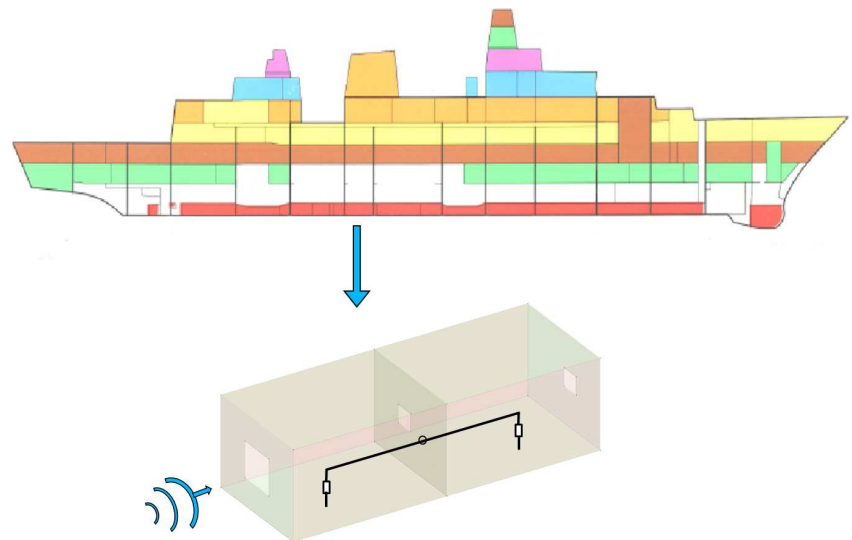

Fig. 1. Abstraction from a ship to a two-room model irradiated by an external interfering source.

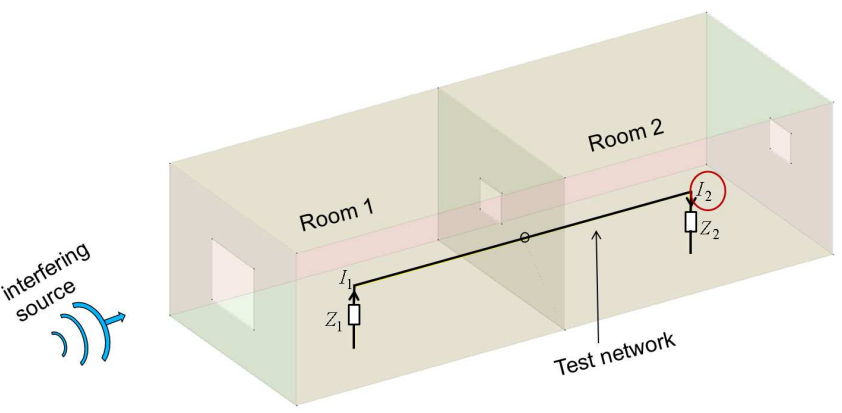

Fig. 2. View of the investigated two-rooms geometry with a test network.

network runs through a small opening at the wall interface between the two rooms. The portion of the test network in room 1 has the length $l_{1}$. The goal is to estimate the induced common mode current at the input of the electronic component connected to the end of the test network in room 2, that is caused by the external interfering source. The structure as well as the test network are electrically large.

\section{Classification of couplings and simplification procedure}

\subsection{Classification of couplings}

The first step consists in making a topological analysis of the system. In this analysis, the possible coupling paths from the source to the target system are identified. This step gives us a visual information of the contribution of the different coupling paths to the overall response of the system leading to the unknown current $I_{2}$. The result of such an analysis is illustrated in Fig. 3. Hereby we neglected contributions due to diffusion effects trough the walls. After identifying those

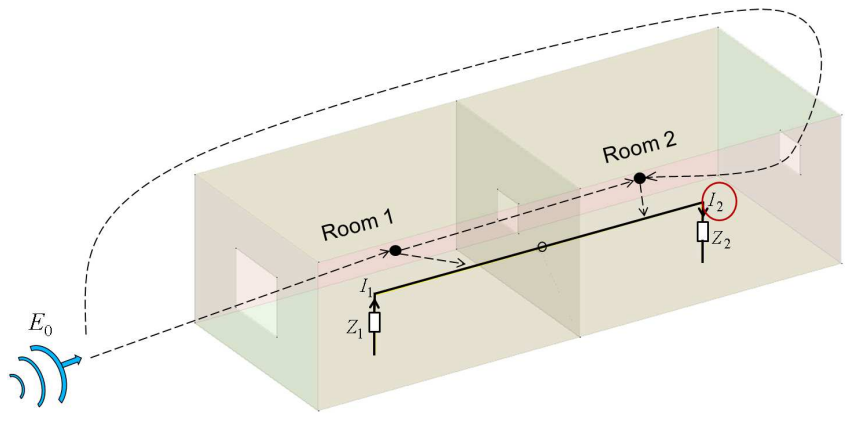

Fig. 3. Identification of possible coupling paths.

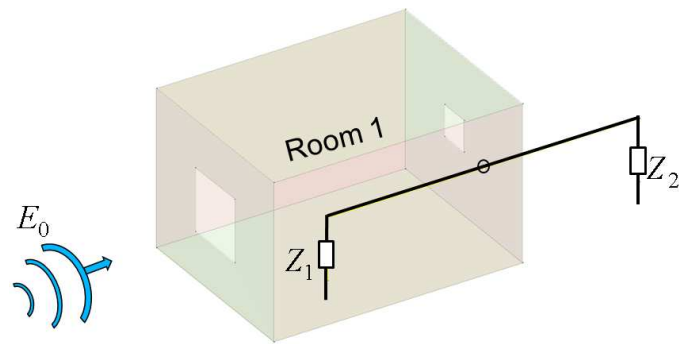

Fig. 4. Disregard of weak coupling paths.

coupling paths we proceed with a ranking of those paths. This ranking takes parameters into account such as the electrical length of apertures, the dimension of gasket opening and also the routing of the network. In case of an electrically very small aperture, we can consider that the contribution of the coupling paths going through this aperture is of second importance and therefore classify this coupling path as a weak one. The electrical dimension of opening for feeding cable through is of importance because it influences the impedance characteristics of the cable and also introduces parasitic capacitive effects when its dimension gets smaller. The routing of the cable also influences the coupling behavior and coupling paths involving small apertures have to be take into account if there are cables running directely in front of those apertures (Parmantier and Degauque, 1996).

\subsection{Simplification procedure}

Using the problem described in Sect. 2 and after applying the ranking procedure described before, it was possible to classify the coupling paths through the apertures of room 2 as weak. The decision was taken according to the electrical dimension of the apertures and the route of the test network. As a result of this, we obtained a simplified geometry where we neglected the influence of room 2 as illustrated in Fig. 4. Before modeling the geometry using a 3-D field simulation software, we needed to apply an impedance transformation on $Z_{2}$. Doing that, we obtained a simplified geometry as 


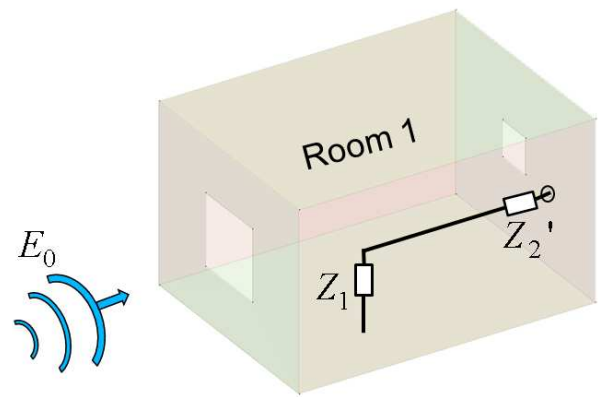

Fig. 5. Reduced equivalent geometry for 3-D-field computation.

shown in Fig. 5, in which the portion of the network previously located in room 2 is transformed with respect to Eq. (1) and is represented by the equivalent impedance $Z_{2}^{\prime}$.

$Z_{2}^{\prime}=Z_{0} \cdot \frac{Z_{2}+Z_{0} \cdot \tanh (\gamma l)}{Z_{0}+Z_{2} \cdot \tanh (\gamma l)}$

In Eq. (1) $Z_{0}$ represents the characteristic impedance of the cable.

It can be obtained by using the formulae (2-5) which are valid for lossless transmission lines.

$Z_{0}=\sqrt{\frac{L^{\prime}}{C^{\prime}}}$

$L^{\prime}$ and $C^{\prime}$ are the per unit length inductance and capacitance and are derived from the transmission line height $h$, the radius $r$ and the material parameters $\mu$ and $\varepsilon$ with the help of the following equations.

$L^{\prime}=\frac{\mu}{2 \pi} \cdot \ln \frac{2(h-r)}{r}$

$C^{\prime}=\frac{\mu \varepsilon}{L^{\prime}}$

The propagation constant is taken from Eq. (5).

$\gamma=\mathrm{j} \omega \sqrt{L^{\prime} \cdot C^{\prime}}$

We neglected the influence of the opening for feeding cable through during this investigation.

\section{Estimation of the induced current}

We used the simplified geometry in Fig. 5 that is less complex compared to the original geometry in Fig. 2, and computed the current $I_{2}^{\prime}$ inside the equivalent impedance $Z_{2}^{\prime}$. Then we made the assumption that the current flowing in

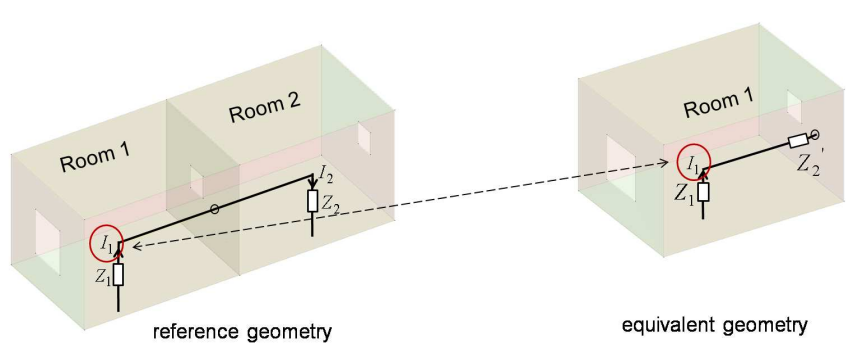

Fig. 6. Equivalence of current in region with strong coupling.

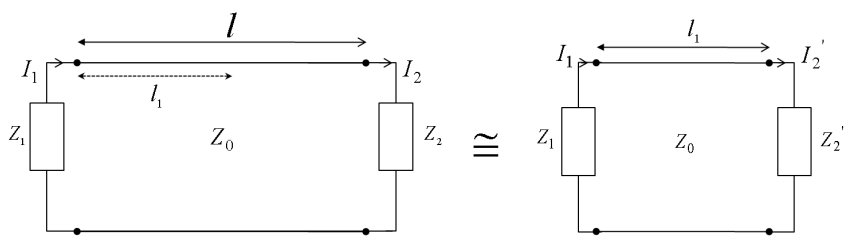

Fig. 7. Transmission line modeling of the equivalence.

the impedance $Z_{1}$ of Fig. 4 should be the same as the one flowing in $Z_{1}$ of Fig. 2. This is shown in Fig. 6. With this assumption and using transmission line theory formulae for the propagation behavior of currents and voltages along a line, the unknown current $I_{2}$ from the reference geometry can be derived from $I_{2}^{\prime}$ (Fig. 7), obtained with the reduced geometry according to Eq. (6).

$I_{2}=I_{2}^{\prime} \cdot \frac{Z_{2}^{\prime} \cdot \sinh \left(\gamma l_{1}\right)+Z_{0} \cdot \cosh \left(\gamma l_{1}\right)}{Z_{2} \cdot \sinh (\gamma l)+Z_{0} \cdot \cosh (\gamma l)}$

After applying our estimation procedure using the reduced geometry of Fig. 5 to compute the current $I_{2}$, we made an exact 3-D-field computation involving the reference geometry of Fig. 2 to appreciate the validity of our estimation procedure.

Looking at the comparison in Fig. 8, we note that there is a good agreement between the exact computation with the reference geometry and our estimation using the reduced geometry. The resonance characteristic is well reproduced.

In industry applications, we can take the magnitude difference between both results into account by adding an extra security margin onto our results. This is particularly true for lower frequencies where the simplification leads to neglect the influence of some late time components of the interfering source. This comparison also allows us to affirm the applicability of our procedure in electrically large multi-room structures. 


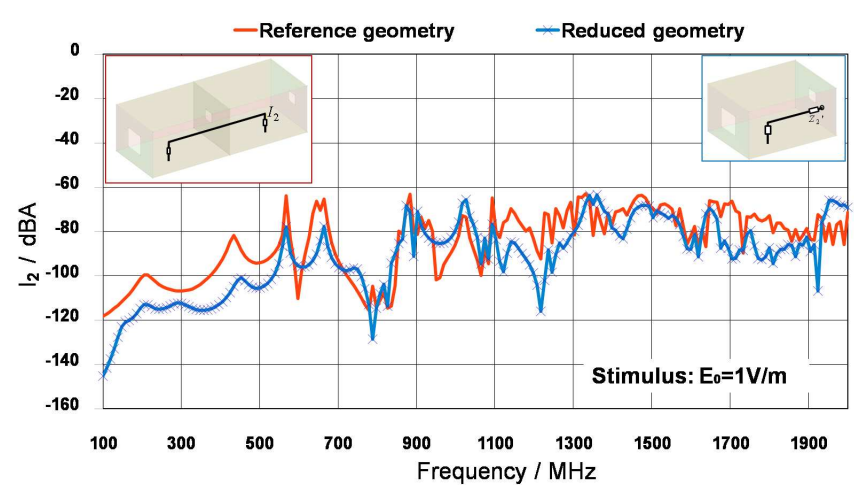

Fig. 8. Comparison between exact computation and estimation using a reduced geometry.

\section{Conclusions}

In this paper we described a methodology which allows a simplification of problems involving multi-rooms structures. We presented necessary conditions based on a topological analysis for the classification of coupling paths as weak or strong, and we could use this ranking to simplify an electrically large multi-room system involving a network going through the sub-volume of the system. With this simplification, an estimation of the coupled disturbances on cables is easier. We showed the applicability of this method using a two-room structure and the advantage of the method which will be more visible when dealing with structures made up of dozen of rooms like in ships. Further optimization can be achieved through a more complex computation of the transmission line parameters and through a consideration of the capacitive effects introduced by the opening for feeding cable through.
Acknowledgement. This work has been financed by the Bundesamt für Wehrtechnik und Beschaffung.

\section{References}

Kanyou Nana, R., Dickmann, S., and Sabath, F.: Electromagnetic field vulnerability of complex systems - an application of EM topology, Adv. Radio Sci., 6, 273-277, 2008, http://www.adv-radio-sci.net/6/273/2008/.

Baum, C. E.: Electromagnetic Topology: A formal approach to the analysis and design of complex electronic systems, Proc. Zurich EMC Symp., 209-214, 1982.

Parmantier, J. P. and Degauque, P.: Topology based modelling of very large systems, Modern Radio science, edited by: Hamelin, J. Oxford University Press, pp. 151-177, 1996.

Tesche, F. M., Ianov, M. V., and Karlsson, T.: EMC Analysis Methods and Computational Models, ISBN 0-471-15573-X, John Wiley \& Sons, Inc., New York, 1996.

Paul, C. R.: Analysis of Multiconductor Transmission Lines, ISBN 0-471-02080-X, John Wiley \& Sons, Includes bibliographical references and index, New York, 1994.

Giri, D. V.: Radiation of impulse-like waveforms with illustrative applications, Ultrawideband and Ultrashort Impulse Signals, Sevastopol, Ukraine, 19-22 September 2004.

EM Software \& Systems-S.A (Pty) Ltd: FEKO Suite 5.4 Online Help, 2008.

Haase, H., Nitsch, J., and Steinmetz, T.: Transmission-Line Super Theory: A new Approach to an Effective Calculation of Electromagnetic Interactions, Radio Science Bulletin, pp. 33-60 No. 307, ISSN 1024-4530, International Union of Radio Science, URSI, c/o University of Gent, 2003. 